\title{
Review of: "A combined generalized Warblet transform and second order synchroextracting transform for analyzing nonstationary signals of rotating machinery"
}

\author{
Nianyin Zeng ${ }^{1}$ \\ 1 Xiamen University
}

Potential competing interests: The author(s) declared that no potential competing interests exist.

This paper aims at overcoming the drawbacks of time-frequency analysis (TFA) techniques in handling strong non-stationary vibration signals. It combines two TFA methods together to enhance concentration, namely GWT-SSET that based on generalized Warblet transform (GWT) and second order synchro extracting transform (SSET) theories. Several quantized indicators are used for evaluation in numerical experiments to show the effectiveness of the proposal. It is concluded that proposed GWT-SSET has potential for practical application. There are some concerns with this study which include following:

1. In many places an abbreviation TFR has occurred for several times whereas without providing the full name, which seems to be short for time-frequency representation.

2. Plenty of advanced TFA methods have been introduced, which mainly concentrate on a better TFR concentration. However, the essence of such improvements remains unspecified. To this end $\square(a)$ Revealing or analyzing why those strategies can bring out better results is helpful for an overall understanding of this work, which can just focus on a few selected classical works. (b) Previous solutions to this problem should be addressed in a systematic manner. Particularly, how proposed method will handle mentioned issues seems not elaborated. (c) Motivation of the combination of GWT and SSET is supposed to be further reasoned, including the rationality and feasibility, which will provide a wider view to conduct relevant research.

3. Many mathematical expressions are used in section theoretical background, and there are some mistakes or confusing issues. (a) While simplifying the equation (5), the expression has become a summation of finite items but in the first line there is an infinite symbol. (b) Equation (9) should be the partial derivative of $\operatorname{STFT}^{\wedge}\{g\}_{-}\{x\}(t, w)$ with respective to $t$ while equation (10) is further derivate based on (9), which generates the second-order derivative. However, the description of equation (9) is wrongly written, which is totally same as it of equation (10).

4. Aiming at the experimental validation part, (a) Whether collected data need pre-processing is not clarified. Different from numerical evaluation, more detail information on processing real data should be added. (b) GWT-SSET is only compared with the baseline method GWT, thus the authors may overstate the practicality of their proposals, which just proves combination with SSET is better. But compared with 
other advanced techniques, is it still recommended to apply GWT-SSET

5. In proposed GWT-SSET, SSET is depicted as a post-processing technique for GWT. According to Table.1, the second Renyi Entropy value (5.60) is calculated by SSET which is better than that of GWT (6.73). (a) The authors make no comments on this point, since SSET already presents a better performance, how to guarantee or measure the positive contributions of applying GWT in the previous stage? (b) Other selected TFA methods for comparison perform even worse than single GWT approach. Hence, their references are suggested to be provided so that one can see whether they are qualified to stand for the state-of-the-art methods.

6. It is also suggested to provide more future directions in the conclusion part. For instance, except for traditional signal processing methods, deep learning techniques have boomed and witnessed a many of successes in various fields. Is it feasible to apply deep learning based time-series analysis method to nonstationary signals? 